\title{
Desempenho de Alagados Construídos Cultivado com Tifton 85 (Cynodon Dactylon
}

\author{
Pers.) \\ Performance of Constructed Wetlands Cultivated with Tifton 85 (Cynodon Dactylon Pers.) \\ Rendimiento de los humedales artificiales cultivados con Tifton 85 (Cynodon Dactylon Pers.)
}

Recebido: 26/04/2021 | Revisado: 30/04/2021 | Aceito: 06/05/2021 | Publicado: 07/05/2021

Maria Fernanda Antunes Collares
ORCID: https://orcid.org/0000-0003-3111-5380
Universidade Federal de Viçosa, Brasil
E-mail: maria.collares@ ufv.br
Fernando Colen
ORCID: https://orcid.org/0000-0001-6039-1240
Universidade Federal de Minas Gerais, Brasil
E-mail: fernandocolenufmg@ @mail.com
Irene Menegali
ORCID: https://orcid.org/0000-0001-5323-4693
Universidade Federal de Minas Gerais, Brasil
E-mail imenegali@ ufmg.br
Rodolpho Cesar dos Reis Tinini
ORCID: https://orcid.org/0000-0003-2052-5368
Universidade Federal de Minas Gerais, Brasil
E-mail: rodolphotinini @ica.ufmg.br
Leonardo França da Silva
ORCID: https://orcid.org/0000-0002-9710-8100
Universidade Federal de Viçosa, Brasil
E-mail: leonardo.silva @ufv.br

\begin{abstract}
Resumo
O presente estudo teve por objetivo avaliar um sistema de alagados construídos (SACs) de fluxo vertical utilizando como meio suporte brita e areia, cultivados com Cynodon dactylon Pers., para o tratamento de águas residuárias de suinocultura. O experimento foi conduzido nos meses de agosto a novembro de 2017 , na área experimental do Instituto de Ciências Agrárias da UFMG. O delineamento experimental foi inteiramente casualizado com três repetições em cada tratamento. Os dados foram submetidos a teste de ANOVA e o teste de comparação de médias com 5\% de significância. Observou-se que para a remoção de DQO, os tratamentos foram diferentes estatisticamente, podendo destacar a elevada eficiência do tratamento com brita + planta chegando a $96 \%$. Na eficiência de remoção de turbidez, sólidos totais, fixos e voláteis entre os tratamentos não houve diferença significativa, mas é possível observar uma redução considerável dos parâmetros avaliados. Para pH a maior média ficou com o tratamento brita + planta e, para a condutividade elétrica a maior média foi do tratamento areia. $\mathrm{O}$ tifton 85 cultivado apresentou boa adaptação ao meio tendo assim um bom crescimento e produção. Com isso, concluiu-se que o sistema de alagados construídos para o tratamento de águas residuárias de suinocultura é eficiente, sendo recomendado o uso de cultivares como o tifton 85 por apresentar potencial nutritivo para a alimentação animal.
\end{abstract}

Palavras-chave: Efluentes; Água de suinocultura; Águas residuárias; Wetlands.

\begin{abstract}
Abstratct
The objective of the present study was to evaluate a vertical flow system (SACs), using crusher and sand, cultivated with Cynodon dactylon Pers., For the treatment of swine wastewater. The experiment was conducted from August to November 2017, in the experimental area of the Institute of Agricultural Sciences of UFMG. The experimental design was completely randomized with three replicates in each treatment. Data were submitted to ANOVA test and the means comparison test with 5\% significance. It was observed that for the removal of COD, the treatments were statistically different, being able to highlight the high efficiency of treatment with gravel + plant reaching $96 \%$. In the turbidity removal efficiency, total, fixed and volatile solids between treatments there was no significant difference, but it is possible to observe a considerable reduction of the evaluated parameters. For $\mathrm{pH}$ the highest average was with the treatment brita + plant and, for the electrical conductivity the highest average was the sand treatment. The cultivated tifton 85 showed good adaptation to the medium thus having a good growth and yield. Therefore, it was concluded that the system of floodwaters built for the treatment of swine wastewater is efficient, being recommended the use of cultivars such as tifton 85 because it is nutritious for animal feed.
\end{abstract}

Keywords: Effluents; Swine water; Wastewater; Wetlands. 


\begin{abstract}
Resumen
El presente estudio tuvo como objetivo evaluar un sistema de humedales construidos (SAC) de flujo vertical utilizando grava y arena como medio de soporte, cultivado con Cynodon dactylon Pers., Para el tratamiento de aguas residuales porcinas. El experimento se realizó de agosto a noviembre de 2017, en el área experimental del Instituto de Ciencias Agrícolas de la UFMG. El diseño experimental fue completamente al azar con tres repeticiones en cada tratamiento. Los datos fueron sometidos a la prueba ANOVA y la prueba de comparación de medias con un 5\% de significancia. Se observó que para la remoción de DQO, los tratamientos fueron estadísticamente diferentes, pudiendo resaltar la alta eficiencia del tratamiento con grava + planta alcanzando el 96\%. En la eficiencia de remoción de turbidez, sólidos totales, fijos y volátiles entre tratamientos, no hubo diferencia significativa, pero es posible observar una reducción considerable en los parámetros evaluados. Para el pH, el promedio más alto fue con el tratamiento de grava + planta y, para la conductividad eléctrica, el promedio más alto fue para el tratamiento de arena. El tifton 85 cultivado mostró una buena adaptación al medio, por lo que tuvo un buen crecimiento y producción. Así, se concluyó que el sistema de ciénaga construido para el tratamiento de aguas residuales porcinas es eficiente y se recomienda el uso de cultivares como tifton 85 por su potencial nutricional para la alimentación animal.
\end{abstract}

Palabras clave: Efluentes. Agua porcina. Aguas residuales. Humedales.

\title{
1. Introdução
}

A poluição ambiental é agravada quando a exploração dos recursos naturais é realizada de forma desordenada e, tende a potencializar seus impactos com o crescimento acelerado da população e a falta dos devidos procedimentos de tratamento e destinação adequados dos resíduos. A demanda pelos recursos hídricos está cada dia mais elevada devido à industrialização, necessidades do setor doméstico e agrícola.

A atividade da suinocultura, no Brasil, é extremamente relevante para a geração de empregos, renda e produção de proteína animal (Zhang, 2020), porém o desenvolvimento acelerado de fazendas de suínos gera consequências ambientais desagradáveis, tendo a necessidade de um tratamento e destinação adequado da mesma (Waki et al., 2018). O despejo de águas residuárias de suínos tem representado uma séria ameaça aos corpos hídricos em áreas rurais e tem recebido atenção cada vez maior em todo o mundo, pois sem os métodos adequados de tratamento de águas residuais, o efluente pode contaminar os recursos hídricos (Duong et al., 2021).

Acredita-se que a má remoção de nutrientes de águas residuais de suinocultura por sistemas convencionais existentes, como fossas sépticas, estabilização de resíduos e lagoas de oxidação, contribuem para o declínio da qualidade da água e alto custo de operação devido às demandas técnicas e de energia (Udom et al., 2018). Com isso, o tratamento das águas residuárias através de sistemas alagados construídos - SACs, por ser uma alternativa de baixo custo de operação e manutenção em comparação com outros sistemas de tratamento de águas residuais biológicas e boa eficiência, no qual, utilizam como meio suporte materiais de uso comum como areia e brita (Afzal et al., 2019; Feng et al., 2020; Li et al., 2020).

Os SACs utilizam-se de plantas cultivadas nos substratos auxiliando no tratamento das águas residuárias, além de harmonizar com a paisagem natural, obter um efluente com nível de purificação desejada para seu reuso ou destinação final de qualidade ideal para serem lançados em corpos hídricos desde que estejam em conformidade com as leis ambientais vigentes (Fia et al., 2015).

A água residual é purificada por filtração do substrato, absorção da planta, degradação do biofilme nas superfícies do substrato e rizosferas das plantas. A funcionalidade dos SACs é fornecida principalmente por micróbios, e as plantas podem promover a realização de funções microbianas (Liu et al., 2020).

Dentre as cultivares empregadas neste sistema, o tifton 85 é uma espécie que possui características de elevada produção, boa capacidade de crescimento e fácil adaptação a condições extremas desde que seu manejo seja correto. Desenvolve também, papel relevante de extração de minerais, estabilização da carga orgânica, redução da turbidez e também contribui no aumentando a vida útil dos SACs (Parde et al.,2021). Além disso, é uma importante forrageira na alimentação e nutrição animal mantendo uma boa qualidade para a produção de carne e leite. 
Os wetlands estão cada vez mais ganhando popularidade devido a um conjunto de recursos valiosos como a remediação de águas residuais em condições variadas, preservação da qualidade do ecossistema, conservação da paisagem e benefícios estéticos (Sharma, et al., 2021). Diante do exposto, objetivou-se com esse trabalho verificar a eficiência dos Sistemas Alagados Construídos - SACs cultivados com tifton 85 (Cynodon dactylon Pers.) no tratamento de águas residuárias da suinocultura, utilizando como substrato brita zero e areia para as condições climáticas do município de Montes Claros-MG.

\section{Metodologia}

O experimento foi conduzido na Fazenda Experimental Professor Hamilton de Abreu Navarro da Universidade Federal de Minas Gerais campus Montes Claros/MG, em uma casa de vegetação coberta por tela plástica (sombrite 70\%) e laterais abertas.

Na primeira fase do experimento foi realizada a montagem dos alagados construídos e a segunda englobou a coleta de dados e análises. Para a montagem das unidades experimentais utilizou-se doze de tudo PVC de $200 \mathrm{~mm}$ com sistema de drenagem de água residuária constituído por um tubo de $20 \mathrm{~mm}$ localizado no fundo dos leitos indo até uma altura de $20 \mathrm{~cm}$ acima da base do leito. O meio suporte utilizado foi brita zero e areia.

Foram montados 12 SACs sendo 6 deles com meio suporte areia e 6 deles com brita zero sendo cada tratamento com três repetições com e sem o capim tifton 85. A água residuária da suinocultura era colocada pela parte superior dos SACs utilizando regadores.

A espécie escolhida para o cultivo foi o capim tifton-85 (Cynodon dactylon Pers) que apresenta boas características de produção e elevada capacidade de crescimento chegando até um metro de altura (MATOS; ABRAHÃO; PEREIRA, 2008). Segundo Teixeira et al. (2018), quanto maior a concentração de nutrientes no efluente tratado, maior é a extração pelas plantas até atingir um ponto máximo, característico de cada cultura.

Foi realizada em triplicata a determinação do índice de vazios para a brita e areia conforme Zanella (2008), tendo como resultado 49,00 e 40,50\% respectivamente. Após o enchimento dos SACs com o meio suporte e a água residuária, as espécies foram plantadas e conduzidas, objetivado uma adaptação da cultivar ao ambiente, o que durou 10 dias. A coleta para as análises foi realizada quinzenalmente em frascos de vidros, os quais foram encaminhados ao laboratório de Tratamento de Resíduos no ICA/UFMG. Foram analisados o potencial hidrogeniônico $(\mathrm{pH})$ através do pHmetro digital, demanda química de oxigênio (DQO), Turbidez, Condutividade elétrica (CE), Sólidos Totais, fixos e voláteis (APHA, 2015).

$\mathrm{O}$ experimento foi conduzido em delineamento inteiramente casualizados onde todos os tratamentos receberam as mesmas condições. Para a avaliação das variáveis, os dados foram submetidos ao teste de Shapiro-Wilk para a normalidade das amostras, de variância através de ANOVA a 5\% de probabilidade e teste de médias Tukey. O programa estatístico utilizado foi o R.

\section{Resultados e Discussão}

\subsection{Demanda Química De Oxigênio (DQO)}

A DBO é usada para medir quanto oxigênio é consumido na decomposição de poluentes orgânicos. OS SACs são considerados eficientes na remoção de uma variedade de poluentes orgânicos e inorgânicos como por exemplo DBO de águas residuais por meio de mecanismos como degradação microbiana, absorção de plantas, oxidação química, adsorção, filtração, precipitação biológica, volatilização e bioestabilização (Lu et al., 2020 \& Vymazal et al.,2020). Os resultados obtidos na análise temporal de DQO estão expressos na Tabela 1. 
Tabela 1 - Valores médios e eficiência.

\begin{tabular}{cccccccccc}
\hline \multicolumn{10}{c}{ Valores médios de DQO } \\
\hline Dias & $\begin{array}{c}\text { Entrada } \\
\left(\mathrm{mg} \mathrm{L}^{-1}\right)\end{array}$ & $\begin{array}{c}\text { Areia } \\
\left(\mathrm{mg} \mathrm{L}^{-1}\right)\end{array}$ & $\begin{array}{c}\text { Eficiência } \\
(\%)\end{array}$ & $\begin{array}{c}\text { Areia + Planta } \\
\left(\mathrm{mg} \mathrm{L}^{-1}\right)\end{array}$ & $\begin{array}{c}\text { Eficiência } \\
(\%)\end{array}$ & $\begin{array}{c}\text { Brita } \\
\left(\mathrm{mg} \mathrm{L}^{-1}\right)\end{array}$ & $\begin{array}{c}\text { Eficiência } \\
(\%)\end{array}$ & $\begin{array}{c}\text { Brita + } \\
\text { Planta } \\
\left(\mathrm{mg} \mathrm{L}^{-1}\right)\end{array}$ & $\begin{array}{c}\text { Eficiência } \\
(\%)\end{array}$ \\
\hline 10 & 467,15 & 340,63 & $27,08 \mathrm{AB}$ & 161,80 & $52,50 \mathrm{~B}$ & 145,98 & $68,75 \mathrm{AB}$ & 142,34 & $69,53 \mathrm{~A}$ \\
18 & 1459,83 & 398,89 & $72,67 \mathrm{AB}$ & 255,77 & $35,88 \mathrm{~B}$ & 409,05 & $71,97 \mathrm{AB}$ & 148,66 & $89,82 \mathrm{~A}$ \\
22 & 2453,13 & 404,17 & $83,52 \mathrm{AB}$ & 109,33 & $72,95 \mathrm{~B}$ & 235,42 & $90,40 \mathrm{AB}$ & 80,67 & $96,71 \mathrm{~A}$ \\
26 & 2850,00 & 452,38 & $84,13 \mathrm{AB}$ & 279,76 & $38,16 \mathrm{~B}$ & 229,76 & $91,94 \mathrm{AB}$ & 213,09 & $92,52 \mathrm{~A}$ \\
\hline \multicolumn{2}{c}{ Média (DP) } & $66,85(27,03) \mathrm{AB}$ & $49,87(17,05) \mathrm{B}$ & $80,76(12,10) \mathrm{AB}$ & $87,15(12,08) \mathrm{A}$ \\
\hline
\end{tabular}

Legenda: AP - areia + planta; BP - Brita + planta.

Fonte: Autores (2017).

Pode-se observar na Tabela 1 que para a remoção de DQO não houve diferença estatística $(\alpha=5 \%)$ nos tratamentos, contudo, destaca-se uma eficiência de $96 \%$ para BP e $91 \%$ brita. Rodrigues (2016) apresentou valores similares de remoção de DQO demonstrando boa eficiência chegando a 96,48 \% e, Matos, Freitas e Lo Monaco (2010) apresentaram resultados com eficiência de $91 \%$ na remoção de DQO.

Matos et al., (2010) e Fia et al., (2016) ambos em seus trabalhos utilizando o substrato brita zero, cultivados com o capim tifton 85 no tratamento de água residuária da suinocultura, obtiveram resultados inferiores de eficiência em relação à remoção de DQO em SACs cultivados, ficando entre 88 a $90 \%$ e, 79 a 82\%, respectivamente, o que pode ser comparado com os resultados encontrados nos SACs com AP e areia somente. Lu et al. (2021) em seu trabalho obteve a remoção de DQO de 53-91,5\% utilizando o cascalho como substrato do meio suporte.

O tratamento BP apresentou a maior média geral para todo o período com 87\%, diferindo estatisticamente dos demais tratamentos (Tabela 1) e, o tratamento AP apresentou a menor média chegando a 49\% de remoção de DQO ( $\alpha=5 \%)$ de acordo com o teste de Tukey podendo observar a eficiência na remoção de DQO. Nos primeiros dias de avaliação a eficiência foi parcialmente baixa, pois o sistema se encontrava em fase de ajustamento, o mesmo foi identificado por FIA et al.,(2010) na fase I do seu experimento. Eficiências de remoção do trabalho de Udom et al. (2018) foram de até 66,53 \% e a eficiência de remoção de DBO alcançada neste estudo é superior à encontrada por Luo et al. (2018) em que encontrou eficiência variando entre $54,8-74,9 \%$ de remoção.

O capim tifton-85 apresentou influências significativas $(\alpha=5 \%)$ no tratamento BP mas não significativa para o tratamento com areia. Matos et al., (2009) verificaram a não influência de plantas nos SACs na remoção de DQO no tratamento de águas residuárias da suinocultura com o substrato brita zero e cultivo de taboa. Entretanto Matos, Freitas e Lo Monaco (2010), verificaram uma menor colmatação dos poros nos SACs cultivados com tifton 85 e substrato brita zero quando comparado aos não cultivados, o que pode ser observado também no presente trabalho. Tal fato pode ser explicado pela relação tamanho de poros no substrato e um maior crescimento radicular, onde as raízes absorveram mais material orgânico, auxiliando desta forma na eficiência do sistema.

Ademais, a Deliberação Normativa COPAM/CERH No 01 de 2008 vigente no Estado de Minas Gerais determina que um sistema de tratamento deve apresentar uma eficiência na remoção de DQO igual ou superior a 70\%. Desta forma, os resultados apresentados neste trabalho demonstram que para o tratamento AP houve instabilidade para remoção de DQO, não podendo suas efluentes permissões para lançamentos em corpos hídricos. 


\subsection{Condutividade Elétrica $(\mathrm{Ce})$}

$\mathrm{Na}$ Tabela 2 são apresentadas as análises de CE ao longo do tempo das amostras coletadas em SACs com diferentes substratos com e sem cultivo de tifton 85. As amostras do tratamento com o substrato areia apresentaram diferença significativa estatisticamente em relação aos demais tratamentos apresentando uma maior média (Tabela 2) e, as amostras com substrato de AP apresentaram menor média no teste de Tukey $(\alpha=0,05)$.

Observa-se também que a CE da entrada foi menor em comparação com os tratamentos, isso pode ser explicado devido uma baixa absorção de íons pelo sistema radicular das plantas. Matos et al., (2009) em seu trabalho utilizando o substrato brita zero, cultivados com o capim tifton 85 no tratamento de água residuária da suinocultura, encontraram resultados diferentes para este parâmetro, onde os efluentes dos SACs apresentaram valores inferiores aos dos afluentes, explicando que a recirculação da água residuária analisada deve ser baixa, sendo que à medida em que se aumenta a recirculação dessa água utilizada na higienização do local tende a ser mais salina e corrosiva podendo prejudicar os desempenhos dos SACs.

Fia et al., (2016) obtiveram resultados semelhantes, e explica que a degradação do material orgânico presente nas águas residuárias da suinocultura podem liberar íons para a solução, podendo haver perdas de água por evapotranspiração aumentando assim a concentração iônica da água residente nos SACs. PICAM et al., (2020) em seu trabalho obteve resultados diferentes em relação a remoção de CE, pois não apresentaram remoção significativa mesmo avaliando duas espécies de diferentes de plantas.

Tabela 2 - Valores médios e eficiência de CE.

\begin{tabular}{|c|c|c|c|c|c|}
\hline \multicolumn{6}{|c|}{ Análise de CE ao longo do tempo (dS m-1) } \\
\hline Dias & Entrada & Areia & Areia + Planta & Brita & Brita + Planta \\
\hline 10 & 3,27 & $4,52 \mathrm{~A}$ & $4,29 \mathrm{~B}$ & $4,45 \mathrm{AB}$ & $4,11 \mathrm{AB}$ \\
\hline 18 & 3,21 & $5,00 \mathrm{~A}$ & $4,40 \mathrm{~B}$ & $4,55 \mathrm{AB}$ & $4,55 \mathrm{AB}$ \\
\hline 22 & 3,71 & $4,99 \mathrm{~A}$ & $4,22 \mathrm{~B}$ & $4,47 \mathrm{AB}$ & $4,33 \mathrm{AB}$ \\
\hline 26 & 3,16 & $4,39 \mathrm{~A}$ & $4,01 \mathrm{~B}$ & $4,33 \mathrm{AB}$ & $4,28 \mathrm{AB}$ \\
\hline \multicolumn{2}{|c|}{ Média (DP) } & $4,73(0,32)$ & $4,23(0,16)$ & $4,45(0,09)$ & $4,32(0,18)$ \\
\hline
\end{tabular}

Fonte: Autores (2017).

\subsection{Potencial Hidrogeniônico (pH)}

Os valores de pH das águas residuais variaram de um mínimo de 6,30 a 8,20 nas águas residuais afluentes a um máximo de 9,80 nas células plantadas e 8,80 no controle (UDOM et al., 2018). Na Tabela 3 são apresentados os valores médios do potencial hidrogeniônico $(\mathrm{pH})$ dos afluentes e efluentes dos SACs estudados. Houve diferença estatística entre os tratamentos BP que apresentaram maior média e AP que apresentaram a menor média (Tabela 3). Os valores encontrados de $\mathrm{pH}$ para os tratamentos foram maiores do que da água residuária da suinocultura.

Houve uma pequena variação do pH médio do afluente, apresentando valores entre 7,3 a 7,6. Matos et al., (2009) em seu trabalho utilizando o substrato brita zero, cultivados com o capim tifton 85 no tratamento de água residuária da suinocultura, relataram dados de aproximadamente 7,0 para águas residuárias de suinocultura. Os valores médios de $\mathrm{pH}$ dos tratamentos encontrados no presente trabalho variaram de 8,25 no tratamento AP à 8,6 no tratamento $\mathrm{BP}$ estando dentro dos valores permitidos para lançamentos, que é de 6 a 9 (CONAMA, 2011).

Fia et al., (2010) em seu trabalho de SACs cultivados com Typha sp. E Alternanthera phyloxeroide no tratamento de água residuária do processamento dos frutos do cafeeiro, explicam que a elevação do $\mathrm{pH}$ nos SACs pode estar ligada a 
liberação de cátions pelo material suporte. Matos et al. (2009) verificaram que no tratamento de águas residuárias de suinocultura em SACs cultivados com tifton 85 , os valores de $\mathrm{pH}$ variaram de 7,0 e 7,1. Apesar da elevação encontrada no $\mathrm{pH}$ médio dos tratamentos, os valores podem ser considerados adequados para o cultivo da espécie vegetal, sendo esta consideração observada também por Fia et al., (2015) em seu trabalho de SACs cultivados com tifton 85 no tratamento da água residuária da suinocultura.

Tabela 3 - Valores médios e eficiência de Ph.

\begin{tabular}{cccccc}
\hline \multicolumn{5}{c}{ Análise ao longo do tempo de pH } \\
\hline Dias & Entrada & Areia & Areia + Planta & Brita & Brita + Planta \\
10 & 7,7 & $8,6 \mathrm{AB}$ & $8,3 \mathrm{C}$ & $8,5 \mathrm{~B}$ & $8,6 \mathrm{~A}$ \\
18 & 7,3 & $8,6 \mathrm{AB}$ & $8,2 \mathrm{C}$ & $8,5 \mathrm{~B}$ & $8,5 \mathrm{~A}$ \\
22 & 7,3 & $8,5 \mathrm{AB}$ & $8,3 \mathrm{C}$ & $8,5 \mathrm{~B}$ & $8,5 \mathrm{~A}$ \\
26 & 7,6 & $8,5 \mathrm{AB}$ & $8,3 \mathrm{C}$ & $8,5 \mathrm{~B}$ & $8,6 \mathrm{~A}$ \\
\hline Média (DP) & & $8,55(0,06)$ & $8,28(0,05)$ & $8,49(0)$ & $8,56(0,06)$ \\
\hline
\end{tabular}

Fonte: Autores.

\subsection{Turbidez}

A turbidez é um parâmetro descritivo das águas no qual representa o grau de interferência com a passagem da luz (VON SPERLING, 2005). De acordo com a Tabela 4, pode-se verificar que os tratamentos não apresentaram diferenças significativas $(\alpha=0,05)$. Porém, a eficiência dos SACs na turbidez é adequada, alcançando a eficiência de $94,87 \%$ no tratamento com AP. Monteiro (2009), em seu trabalho com SACs cultivados em policultura no tratamento de água cinza, verificou uma eficiência média de $92 \%$ na redução da turbidez, sendo explicada através da efetividade da filtração dos SACs.

Freitas (2006) cultivando taboa (Typha latifólia L.), Alternanthera philoxeroides (Mart.) Griseb. e capim tifton - 85 (Cynodon dactyylon Pers.) em SACs no tratamento de águas residuárias da suinocultura, não observou tendência clara de alteração de turbidez, ficando a turdidez de seus efluentes em torno de 20 UNT sendo a turbidez do afluente em torno de 50 a 60 UNT, o que pode ser explicado devido ao desprendimento de partículas orgânicas retidas no filme biológico. Além disso, no presente trabalho o capim tifton 85 cultivado não apresentou diferença na remoção da turbidez, visto também por FREITAS (2006).

Tabela 4 - Valores médios e eficiência de turbidez.

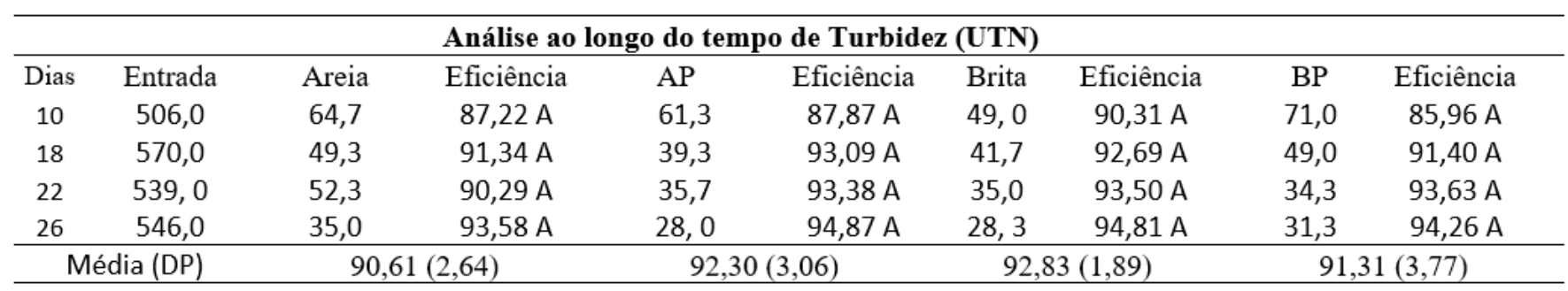

Fonte: Autores.

\subsection{Sólidos}

Os sólidos são usados como um parâmetro de qualidade de águas residuais para avaliar a eficácia de áreas úmidas construídas para a remoção de matéria orgânica e para quantificar o grau de poluição em muitos efluentes de águas residuais industriais (UDOM et al., 2018). 
Nas Tabelas 5, 6 e 7 são apresentadas as séries temporais e eficiências dos sólidos totais (ST), sólidos fixos (SF) e sólidos voláteis (SV), respectivamente das amostras coletadas nos SACs. As concentrações de ST não apresentaram diferenças estatísticas $(\alpha=0.05)$ entre os tratamentos. Os melhores resultados de eficiência na remoção dos ST foram dos SACs com substratos de brita e BP chegando a 67,92\%, semelhante ao que Salati, et al., (2003) encontraram tendo como meio suporte brita e bambu. As plantas têm efeito positivo na remoção de ST, reduzindo a velocidade da água e incentivando a filtração e a biodegradação na rede de raízes entrelaçadas e entrelaçadas (Udom et al., 2018).

A precipitação pluviométrica, as partículas existentes no material utilizado como meio suporte e a baixa remoção dos sólidos dissolvidos, podem ter influenciado nas concentrações dos sólidos totais (Rodrigues, 2016). Freitas (2006), em seu trabalho de SACs para o tratamento da água residuária da suinocultura, afirma que a variação dos sólidos totais é devido à instabilidade, ou variabilidade dos afluentes.

Para os SF as variações médias de eficiência ficaram entre 13,63 \% e 54,54 \%, destacando o substrato brita por apresentar a maior eficiência mesmo que não significativa $(\alpha=0.05)$ e a menor média com areia. Segundo Monteiro (2009), o aumento dos sólidos fixos é esperado, referindo especialmente ao aumento de sais minerais que concentram-se nos SACs. A remoção de ST é muito eficaz em SACs de fluxo vertical devido aos processos físicos de sedimentação, filtração e precipitação química e absorção fornecida pelo meio do pantanal (Udom et al., 2018)

Nos SV as variações não apresentaram diferenças significativas $(\alpha=0.05)$ apresentando médias pouco diferentes ao longo do tempo, ficando as variações de eficiência com a maior média $84,52 \%$ nos tratamentos Areia, Brita e Brita com planta e a menor média 39,58\% no tratamento Brita com planta.

A despeito de não haver diferença estatística entre os tratamentos, evidencia-se que as maiores eficiências foram na presença da cultivar Tifton 85, o que Queiroz et al. (2004) também observaram em seu experimento no tratamento de águas residuárias da suinocultura cultivadas em quatro espécies de forrageiras, sendo elas, quicuio da Amazônia, braquiária, coastcross e tfton 85 , sendo uma cultivar importante para aumentar a remoção de nutrientes e material orgânico no meio de cultivo. Quando as águas residuais com alto teor de ST são descarregadas em águas superficiais ou subterrâneas, esses sólidos dissolvidos podem representar uma fonte significativa de poluição (Udom et al., 2018).

Tabela 5 - Valores médios e eficiência de Sólidos Totais.

\begin{tabular}{|c|c|c|c|c|c|c|c|c|c|}
\hline \multicolumn{10}{|c|}{ Análise ao longo do tempo Sólidos Totais $\left(\mathrm{gL}^{-1}\right)$} \\
\hline Dias & Entrada & Areia & Eficiência & $\mathrm{AP}$ & Eficiência & Brita & Eficiência & BP & Eficiência \\
\hline 10 & 0,5300 & 0,1833 & $65,40 \mathrm{~A}$ & 0,3367 & $36,47 \mathrm{~A}$ & 0,1700 & $67,92 \mathrm{~A}$ & 0,1700 & $67,92 \mathrm{~A}$ \\
\hline 18 & 0,3900 & 0,2000 & $48,71 \mathrm{~A}$ & 0,2000 & $48,71 \mathrm{~A}$ & 0,1667 & $57,26 \mathrm{~A}$ & 0,2200 & $43,58 \mathrm{~A}$ \\
\hline 22 & 0,3700 & 0,2700 & $27,02 \mathrm{~A}$ & 0,1800 & $51,35 \mathrm{~A}$ & 0,2100 & $43,24 \mathrm{~A}$ & 0,2367 & $36,03 \mathrm{~A}$ \\
\hline 26 & 0,5700 & 0,2300 & $59,64 \mathrm{~A}$ & 0,2233 & $60,81 \mathrm{~A}$ & 0,2200 & $61,40 \mathrm{~A}$ & 0,2567 & $54,97 \mathrm{~A}$ \\
\hline \multicolumn{2}{|c|}{ Média (DP) } & \multicolumn{2}{|c|}{$50,20(16,93)$} & \multicolumn{2}{|c|}{$49,34(10,03)$} & \multicolumn{2}{|c|}{$57,46(10,44)$} & \multicolumn{2}{|c|}{$50,63(13,91)$} \\
\hline
\end{tabular}

Fonte: Autores.

Tabela 6 - Valores médios e eficiência de Sólidos Fixos.

\begin{tabular}{rrrrrrrrrc}
\hline \multicolumn{8}{c}{ Série Temporal Sólidos Fixos $\mathbf{( g L}^{-1}$ ) } \\
\hline Dias & Entrada & Areia & Eficiência & AP & Eficiência & Brita & Eficiência & BP & Eficiência \\
10 & 0,2500 & 0,1400 & $44,00 \mathrm{~A}$ & 0,2100 & $16,00 \mathrm{~A}$ & 0,1267 & $49,33 \mathrm{~A}$ & 0,1267 & $49,33 \mathrm{~A}$ \\
18 & 0,2300 & 0,1700 & $26,08 \mathrm{~A}$ & 0,1667 & $27,53 \mathrm{~A}$ & 0,1333 & $42,02 \mathrm{~A}$ & 0,1233 & $46,37 \mathrm{~A}$ \\
22 & 0,2200 & 0,1900 & $13,63 \mathrm{~A}$ & 0,1233 & $43,93 \mathrm{~A}$ & 0,1367 & $37,87 \mathrm{~A}$ & 0,1533 & $30,30 \mathrm{~A}$ \\
26 & 0,3300 & 0,1633 & $50,50 \mathrm{~A}$ & 0,1567 & $52,52 \mathrm{~A}$ & 0,1500 & $54,54 \mathrm{~A}$ & 0,1633 & $50,50 \mathrm{~A}$ \\
\hline
\end{tabular}

Fonte: Autores (2017). 
Tabela 7 - Análise ao longo do tempo com valores médios e eficiência de Sólidos Voláteis.

\begin{tabular}{|c|c|c|c|c|c|c|c|c|c|}
\hline \multicolumn{10}{|c|}{ Análise ao longo do tempo Sólidos Voláteis $\left(\mathrm{gL}^{-1}\right)$} \\
\hline Dias & Entrada & Areia & Eficiència & AP & Eficiência & Brita & Eficiência & BP & Eficiência \\
\hline 10 & 0,2800 & 0,0433 & $84,52 \mathrm{~A}$ & 0,1267 & $54,76 \mathrm{~A}$ & 0,0433 & $84,52 \mathrm{~A}$ & 0,0433 & $84,52 \mathrm{~A}$ \\
\hline 18 & 0,1600 & 0,0300 & $81,25 \mathrm{~A}$ & 0,0333 & $79,17 \mathrm{~A}$ & 0,0333 & $79,17 \mathrm{~A}$ & 0,0967 & $39,58 \mathrm{~A}$ \\
\hline 22 & 0,1500 & 0,0800 & $46,67 \mathrm{~A}$ & 0,0567 & $62,22 \mathrm{~A}$ & 0,0733 & $51,11 \mathrm{~A}$ & 0,0833 & $44,44 \mathrm{~A}$ \\
\hline 26 & 0,2400 & 0,0667 & $72,22 \mathrm{~A}$ & 0,0667 & $72,22 \mathrm{~A}$ & 0,0700 & $70,83 \mathrm{~A}$ & 0,0933 & $61,11 \mathrm{~A}$ \\
\hline Média & & \multicolumn{2}{|c|}{$71,16(17,14)$} & \multicolumn{2}{|c|}{$67,09(10,77)$} & \multicolumn{2}{|c|}{$71,41(14,66)$} & \multicolumn{2}{|c|}{$57,41(20.28)$} \\
\hline
\end{tabular}

Fonte: Autores (2017).

\subsection{Produtividade Do Capim Tifton 85}

Na Tabela 8 é apresentada a produtividade em matéria seca do capim tifton 85 nos SACs com meio suporte brita e areia. Pode-se observar que o capim tifton 85 desenvolveu mais no substrato areia obtendo maior produtividade. A parcela BP3 não foi possível observar valores por ser uma parcela perdida, onde a cultivar não se desenvolveu. A produtividade encontrada no presente trabalho foi de $6,17 \mathrm{tha}^{-1}$ para a maior média da areia e $1,34 \mathrm{t} \mathrm{ha}^{-1}$ para a maior média do substrato brita.

Tabela 8 - Produtividade do capim Tifton 85 .

\begin{tabular}{ccccccc}
\hline \multicolumn{7}{c}{ Produtividade Tifton $85(\mathrm{~g})$} \\
\hline Massa Úmido & 84,1940 & 5,7314 & 16,4525 & 19,2065 & 0,6417 & - \\
Massa Seca & 19,3765 & 1,0397 & 3,9688 & 4,2061 & 0,0549 & - \\
& & & & & AP2 & \\
$\mathrm{kg} \mathrm{ha}^{-1}$ & 6167,73 & 330,947 & 1263,31 & 1338,8432 & 17,4752 & - \\
\hline \hline
\end{tabular}

Fonte: Autores (2017)

Queiroz et al., (2004), obtiveram resultados diferentes com produtividade de 17,7 t ha ${ }^{-1}$ de tifton 85 quando cultivado em SACs com água residuária de suinocultura. Matos, Abrahão e Pereira (2008) encontraram resultados de produtividade de 15,4 t ha ${ }^{-1}$ no desempenho agronômico de capim tifton 85 em SACs utilizados no tratamento de água residuária de laticínios.

Observa-se que a produtividade do capim tifton 85 foi baixa quando comparada a esses autores. Isso pode ser explicado devido ao pouco tempo de cultivo no presente trabalho, que foi de aproximadamente 25 dias, enquanto Queiroz et al., (2004) e Matos, Abrahão e Pereira (2008) trabalharam com um período de aproximadamente 4 meses. A parcela BP 3 foi considerada como perdida, pois o capim não resistiu. Picam et al. (2020) em seu trabalho com diferentes espécies de plantas, não observou diferença na altura das plantas ao longo do experimento nos SCAs cultivados.

\section{Considerações Finais}

Conclui-se que houve boa eficiência nos SACs cultivados com tifton 85 no tratamento de águas residuárias da suinocultura, pois observa-se uma maior remoção de DQO no SACs com meio suporte brita + planta, porém o tifton 85 se desenvolveu melhor nos SACs com meio suporte areia.

Os valores médios de $\mathrm{pH}$ encontraram-se dentro das condições padrões e a remoção da turbidez apresentou boa eficiência principalmente nos SACs com meio suporte areia + planta. Diante disso, recomenda-se o uso dos SACs com a cultivar tifton 85 com meio suporte areia, pois mesmo não apresentando a maior eficiência na remoção de DQO e apresentando valor inferior de eficiência quando comparado com o SACs de meio suporte brita + planta, os SACs cultivados com substrato 
areia apresentam uma maior eficiência turbidez, além de uma maior produção do capim tifton 85 sendo este nutritivo para alimentação animal. Ademais, como possibilidade de estudos futuros, avaliar a remoção de poluentes comparando a produtividade do capim Tifton com outras espécies plantadas. Além disso, analisar outros tipos de SACs como os verticais e comparar a remoção de poluentes e estudar possíveis fatores para evitar o entupimento das saídas dos SACs.

\section{Referências}

Afzal, M., et al (2019). Floating treatment wetlands as a suitable option for large-scale wastewater treatment. Nat. Sustain., 2.

Apha (AMERICAN PUBLIC HEALTH ASSOCIATION) (2015). Standard methods for the examination of water and wastewater. 22.

Conama - Conselho Nacional do Meio Ambiente - RESOLUÇÃO N 430, DE 13 DE MAIO DE 2011 - Dispõe sobre as condições e padrões de lançamento de efluentes, complementa e altera a Resolução n ${ }^{\circ}$ 357, de 17 de março de 2005, do Conselho Nacional do Meio Ambiente-CONAMA. <http://www.mma.gov.br/port/conama/legiabre.cfm?codlegi=646>.

Dong, L., et al (2021). Organics and nutrient removal from swine wastewater by constructed wetlands using ceramsite and magnetite as substrates. Journal of Environmental Chemical Engineering, 9 (1).

Feng, L., et al (2020). Can biochar application improve nitrogen removal in constructed wetlands for treating anaerobically-digested swine wastewater? Chem. Eng. J., 379.

Fia, R. et al.(2010). Desempenho de sistemas alagados no tratamento de águas residuárias do processamento dos frutos do cafeeiro. Revista brasileira de engenharia agrícola e ambiental, 4(12).

Fia, F. R. L., et al. (2017). Effect of vegetation in constructed wetlands treating swine wastewater. Eng. Sanit. Ambient. 22(2). http://dx.doi.org/10.1590/s141341522016123972 .

Fia, R. L., et al (2015). Influência da carga de nutrientes e da espécie cultivada na remoção de K, Na, Cu e Zn da água residuária da suinocultura tratada em sistemas alagados construídos - Ambiente \& Água - An Interdisciplinary Journal of Applied Science, 10(3).

Freitas, W. da S. (2006). Desempenho de Sistemas Alagados construídos, cultivados com diferentes espécies vegetais, no tratamento de águas residuárias da suinocultura. Tese (Doutorado) - Pós- Graduação em Engenharia Agrícola, Universidade Federal de Viçosa, Viçosa.

Li, S., et al (2020). Microalgal and duckweed based constructed wetlands for swine wastewater treatment: a review. Bioresour. Technol., 318 (2020).

Liu, F., et al (2020). Performance of different macrophytes in the decontamination of and electricity generation from swine wastewater via an integrated constructed wetland-microbial fuel cell process. Journal of Environmental Sciences, 89.

Lu D., et al (2021). Organics and nutrient removal from swine wastewater by constructed wetlands using ceramsite and magnetite as substrates. Journal of Environmental Chemical Engineering, 9, (1).

Lu, J., et al (2020). Recent advances in the enhanced nitrogen removal by oxygen-increasing technology in constructed wetlands. Ecotoxicol. Environ. Saf., 205.

Luo, P., et al (2018). Evaluating organics removal performance from lagoon-pretreated swine wastewater in pilot-scale three-stage surface flow constructed wetlands, Chemosphere, 211.

Matos, A. T., Abrahão, S. S., \& Pereira, O. G. (2008). Desempenho agronômico de capim tifton 85 (cynodon spp) cultivado em sistemas alagados construídos utilizados no tratamento de água residuária de laticínios. Revista Ambi-Água. 3(1).

Matos, M. P., Von Sperling, M., Matos, A. T., \& Passos, R. G. (2015). Uso de traçador salino para avaliação da colmatação e dascondições hidrodinâmicas em sistemas alagados construídos de escoamento horizontal subsuperficial. Engenharia Agrícola, 35(6). http://dx.doi.org/10.1590/1809-4430-Eng.Agric. v35n6p1137-1148/2015

Matos, A. T., Freitas, W. S., Fia, R., \& Matos, M. P. (2009) Qualidade do efluente de sistemas alagados construídos utilizados no tratamento de águas residuárias da suinocultura visando seu reuso. Engenharia na Agricultura, 17.

Matos, A. T., Freitas, W. S., \& Lo Monaco, P. A. V. (2010). Eficiência de sistemas alagados construídos na remoção de poluentes de águas residuárias da suinocultura. Ambi-Agua, 5(2).

Matos, A. T., Freitas, W. S., Brasil, M. S., \& Borges, A. C. (2010b). Influência da espécie vegetal cultivada nas condições redox de sistemas alagados construídos. Engenharia Agrícola, 30(3).

Mendonça, H. V. De. (2011). Avaliação da eficiência de sistemas alagados construídos em operação por bateladas no tratamento de efluentes da indústria de laticínios. Dissertação (Mestrado em Ecologia) Universidade Federal de Juiz de Fora, Juiz de Fora.

Monteiro, R. C. De M. (2009). Viabilidade técnica do emprego de sistemas tipo "wetlands" para tratamento de água cinza visando o reuso não potável. Dissertação (Mestrado Engenharia hidráulica e sanitária) - Escola Politécnica da Universidade de São Paulo.

Parde, D., et al (2021) A review of constructed wetland on type, treatment and technology of wastewater. Environmental Technology \& Innovation, 21. 
Research, Society and Development, v. 10, n. 5, e59910515518, 2021

(CC BY 4.0) | ISSN 2525-3409 | DOI: http://dx.doi.org/10.33448/rsd-v10i5.15518

Pincam T., et al (2020). Growth performance of tropical wetland species (Cyperus involucratus Rottb. and Thalia geniculata L.) in anaerobic digester effluent and their water treatment efficiency, Ecological Engineering, 143.

Queiroz, F. M., Matos, A. T., Pereira, O. G., Oliveira, R. A., \& Lemos, A. L. (2004). Características químicas do solo e absorção de nutrientes por gramíneas em rampas de tratamento de águas residuárias da suinocultura. Revista Engenharia na Agricultura, 12(2).

Rodrigues, M. V. C. (2016). Wetland construído de fluxo vertical empregado no tratamento de esgoto de um restaurante universitário. Trabalho de Conclusão de Curso (Bacharelado em Engenharia Ambiental). - Curso de Engenharia ambiental - Universidade Tecnológica Federal do Paraná, Londrina.

Salati, E., et al., (2003). Utilização de sistemas de wetlands construídas para tratamento de águas. São Paulo, Biológico 65 (1-2).

Sharma, R., et al (2021). Application of floating treatment wetlands for stormwater runoff: A critical review of the recent developments with emphasis on heavy metals and nutrient removal. Science of The Total Environment,777.

Silva, S. C. (2007). "Wetlands Construídos” de Fluxo Vertical com Meio Suporte de Solo Natural Modificado no Tratamento de Esgotos Domésticos. Tese de Doutorado em Tecnologia Ambiental e Recursos Hídricos, Departamento de Engenharia Civil e Ambiental, Universidade de Brasília, Brasília, DF, 205.

Teixeira D., Matos A. T., Matos M. P., Vieira D., Araújo E. D., \& Ferraz L. A. (2018). The influence of plant roots on the clogging process and the extractive capacity of nutrients/pollutants in horizontal subsurface flow constructed wetlands. Ecological Engineering, 120(54).

Udom J., C. C. Mbajiorgu, E. O., \&Oboho (2018). Development and evaluation of a constructed pilot-scale horizontal subsurface flow wetland treating piggery wastewater. Ain Shams Engineering Journal, 9 (4).

Von Sperling, M. (2005) Introdução à qualidade das águas e o tratamento de esgotos- 3. ed. Belo Horizonte: Departamento de Engenharia Sanitária e Ambiental, Universidade Federal de Minas Gerais, 28.

Vymazal J., Sochacki A., Fučík P., Šereš M., Kaplická M., Hnátková T., \& Chen Z. (2020). Constructed wetlands with subsurface flow for nitrogen removal from tile drainage. Ecol. Eng., 155 (2020),

Waki M., T. Yasuda, Y. Fukumoto, F. Béline, A. \& Magrí (2018). Treatment of swine wastewater in continuous activated sludge systems under different dissolved oxygen conditions: reactor operation and evaluation using modelling. Bioresour. Technol., 250 (2018).

Zanella, L., (2008) Plantas ornamentais no pós-tratamento de efluentes sanitário: wetlands-construídos utilizando brita e bambu como suporte.189p. Tese (Doutorado) - Faculdade de Engenharia Civil, Arquitetura e Urbanismo, Universidade Estadual de Campinas, Campinas.

Zhang H. (2020). Development of world pig industry in 2019 and its market trends in 2020. Swine Ind. Sci., 37 (2020). 